\title{
Abdominal Cystic Lymphangioma in Adults
}

\author{
K Elatiqi*1, Z Belhadj ${ }^{1}$, MA Bicane ${ }^{2}$, I Haraki ${ }^{3}$, B Finech ${ }^{2}$, K Krati $^{3}$, M Ouali Idrissi ${ }^{1}$ and N Cherif Idrissi Ganouni $^{1}$ \\ ${ }^{1}$ Department of Radiology, Marrakesh \\ ${ }^{2}$ Department of Surgery, Marrakesh
}

${ }^{3}$ Department of Gastro-entérology, Marrakesh

Received: 制: November 29, 2018; Published: 制: December 11, 2018

*Corresponding author: K Elatiqi, Department of Radiology, Marrakesh

\begin{abstract}
Summary
Cystic lymphangioma is a benign vascular tumor originating from the lymphatic pathways, mainly seen in children. This pathology has various locations dominated by the cranio-facial, cervical and axillary regions. Abdominal localization is rare, accounting for $7 \%$ of abdominal cysts in adults. We report the case of a 39-year-old patient with a left ectopic testicle, who consulted for chronic abdominal pain with a normal clinical examination. Ultrasound and CT were performed, showing a cystic intra-abdominal mass suggesting a mesenteric cystic lymphangioma extended to retroperitoneum. Surgical exploration found a cystic mass of the pancreas, extended to the mesentery and proximal jejunum, encompassing the mesenteric pedicle. The diagnosis of cystic lymphangioma was confirmed by histology. In adults, the mesenteric and retroperitoneal involvement are estimated to be less than $1 \%$ of cases. Clinical presentation can be very variable, so here comes the role of imaging in guiding the diagnosis, in the preoperative assessment and in the follow-up. The final diagnosis is made by histology.
\end{abstract}

\section{Introduction}

First described in 1913 by Koch, the cystic lymphangioma is a benign vascular tumor of the lymphatic pathways more common in children, suggesting a malformitive origin [1]. The majority of cystic lymphangiomas occur at the cervico-axillary region. However, intra-abdominal locations such as the mesentery, retroperitoneum and epiploon are possible, but less common, representing about $5 \%$ of cases [2]. We report a case of pacreatic cystic lymphangioma extended to the mesentery with an emphasis on the role of imaging in leading to diagnosis.

\section{Case Report}

We report the case of a 39-year-old patient, known to have an ectopic left testicle, who suffered from a chronic abdominal pain with no general condition deterioration. The clinical examination was normal. An abdominal ultrasound was performed showing a multiloculated cystic mass of the left flank, with anechoic content, non-vascularized on color Doppler, extended to the pelvis (Figure 1). The inguino-scrotal ultrasound revealed a morphologicaly normal ectopic left testicle (inguinal). Abdominal CT was performed showing an extensive cystic mass along the mesentery, encompassing the divisional branches of the superior and inferior mesenteric vessels, the terminal portion of the abdominal aorta and the origin of its divi sional branches, which remain permeable, evoking a cystic lymphangioma. This mass had an intimate contact with the tail of the pancreas and the jejunum at the angle of Treitz (Figures $2 \& 3$ ). The surgical exploration found a pancreatic cystic mass extended to the mesentery and proximal jejunum and encompassing the mesenteric pedicle. As a result, the resection could not be complete leaving a residue near the mesenteric pedicle. In the anatomopathological analysis, the macroscopic and histological aspect was compatible with a cystic lymphangioma (Figure 4).

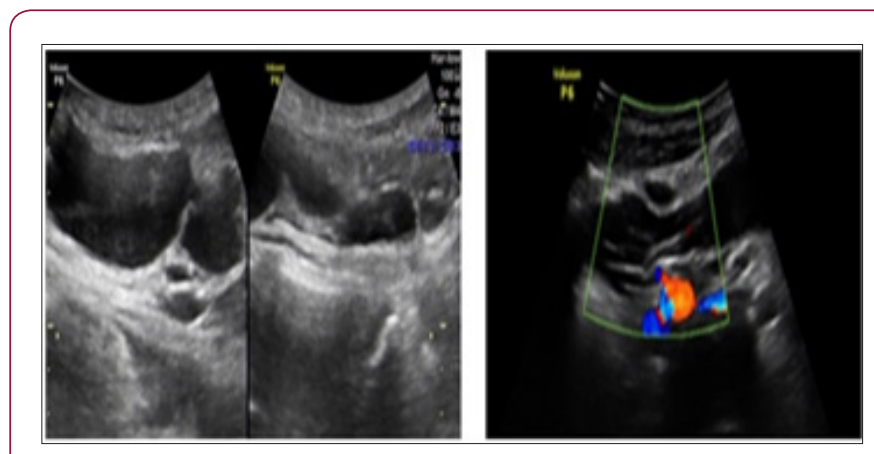

Figure 1: Abdominal ultrasound showing a multiloculated non- vascularized cystic mass. 

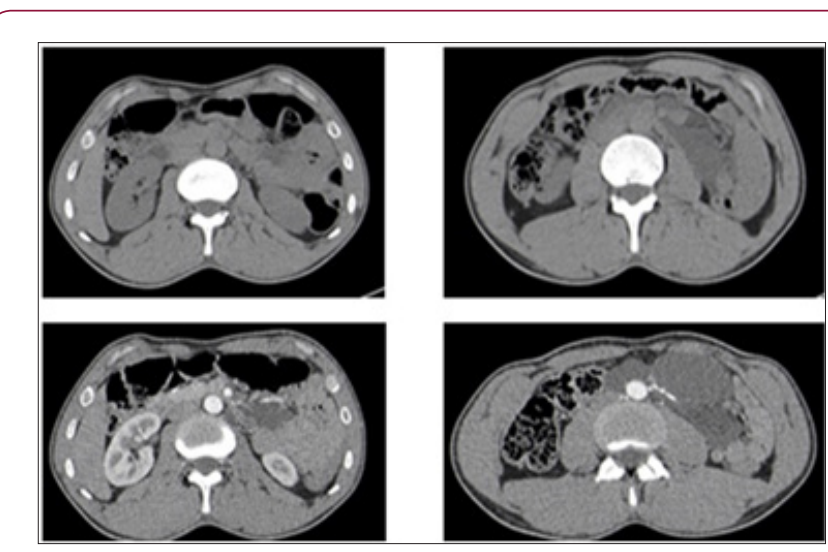

Figure 2: Axial abdominal CT with and without contrast showing a cystic mass of the left flank encompassing mesenteric vessels.

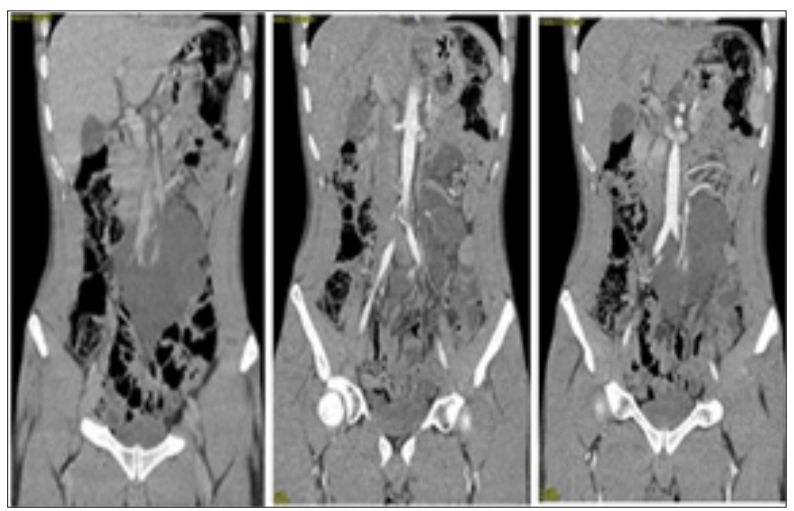

Figure 3: Coronal abdominal CT with and without contrast showing the vascular encompassing.

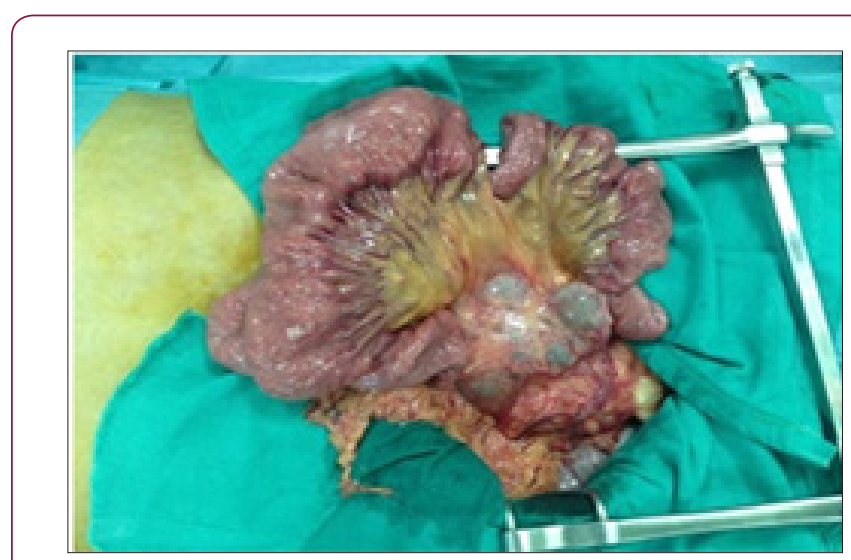

Figure 4: Intraoperative image of the cystic lesion.

\section{Discussion}

The mesentery is a rare localization of cystic lymphangioma estimated at $1 / 100,000$ in adults and $1 / 20,000$ in children [3]. Pancreatic localization is even rarer. Its physio pathogenesis remains unclear, but can be explained by two theories:

\section{Malformative Theory}

In this case, the cystic lymphangioma arises from a disorder of embryogenesis due to a lack of connection between a group of lymphatic chains and the venous system leading to the isolation of lymphatic capillaries, their dilatation then the development of multiple cysts [3].

\section{Acquired Theory}

Especially in adults, cystic lymphangioma occurs in the aftermath of an obstruction of the lymphatic vessels related to a secondary cause (inflammation, trauma or degeneration) [3]. This theory is more and more abandoned [4].

According to the 2018 classification of vascular anomalies, the cystic lymphangioma is considered a lymphatic malformation (simple vascular malformation IIa) [5]. The abdominal cystic lymphangioma preferentially affects the mesentery and retroperitoneum, because of the richness of their lymphatic elements [6]. However, it can reach other intra-abdominal organs (spleen, pancreas, kidney, etc.) [4].

The nonspecific and polymorphic clinical presentation of cystic lymphangioma is related to the volume, the localization and the types of complications it can cause. It can be revealed by the palpation of an abdominal mass, by an abdominal pain, a fever, a hematemesis, a volvulus, etc. In our case, the patient suffered from a chronic abdominal pain. There is a particularly rare clinical form of an abdominal cystic dissemination mimicking peritoneal carcinomatosis, called peritoneal cystic lymphangiomatosis [3].

The ultrasound appearance varies depending on the size of the cubicles. For macrocystic forms, it is a well-defined lesion, uni or multi-cell, with anechoic content separated by very fine fibrous septas. The content may be echoic in case of bleeding or infection. The arterial flow is poor with high resistivity indexes at the septas, without recordable flow within the cubicles. For the micro-cystic forms, the cubicles are very small (infra-millimetric), the mass appears pseudo-tissular, heterogeneously hyperechoic with a posterior reinforcement.

CT is an excellent complementary diagnostic tool. It shows a homogeneous hypodense (cystic) tumor, with thin walls, not enhanced by contrast. The density of the intra-cystic fluid can vary according to the contents which can be serous, chylous or hemorrhagic [3]. MRI is only used as a second-line exam, it allows a more precise study of the anatomical relationships with the neighboring structures.

According to Losanoff and Kjossev, a classification based on the morphotype of the lesion is retained to optimize the surgical approach [7]:

a) The "type 1" is pediculate with a risk of torsion, volvulus : The resection is easy.

b) The "type 2" is sessile, less mobile and may require sacrifice. 
c) The "type 3" involves retroperitoneal extension (sometimes affecting vital structures), making total excision impossible. That was the case for our patient.

d) The "type 4" corresponds to an extensive lesion affecting multiple organs [7].

The differential diagnosis is made with all intra-abdominal cystic lesions: Hydatid cyst, Cystadenoma / cystadenocarcinoma serous or mucinous mesentery, Mesenteric mesothelial cyst, Lymphocele, Dermoid cyst, Hemangioma, Hematoma, Appendicular mucocele, Ovarian cyst etc.

The definitive diagnosis is made by histology [3]. In case of accidental discovery, therapeutic abstention with regular monitoring is recommended if the patient is asymptomatic. Spontaneous regression can be seen in 1.6 to $16 \%$ of cases. Regarding the high risk of progression (volume increase, appearance of complications), surgical excision is the classic approach. It must be total to avoid the recurrence (by laparotomy or laparoscopy) and the most conservative for the organs since it is a benign lesion [6]. The recurrence rate is estimated at $40 \%$ after incomplete resection and at $17 \%$ after macroscopically complete resection. Aspiration of the cyst content with or without sclerosant injection is a potential therapeutic alternative [6].

ISSN: 2574-1241

DOI: 10.26717/BJSTR.2018.11.002174

K Elatiqi. Biomed J Sci \& Tech Res

(C) (P) This work is licensed under Creative

Submission Link: https://biomedres.us/submit-manuscript.php

\section{Conclusion}

Abdominal cystic lymphangioma is a rare condition in adults. The clinical symptomatology is very variable. Imaging plays a key role in guiding the diagnostic, the preoperative assessment and the follow-up. The final diagnosis is made by histology.

\section{References}

1. Gachabayov M, Kubachev K, Abdullaev E, Babyshin V, Neronov D et al. (2016) A huge cystic retroperitoneal lymphangioma presenting with back pain. Case Rep Med pp.1618393.

2. Bhavsar T, Saeed Vafa D, Harbison S, Inniss S (2010) Retroperitoneal cystic lymphangioma in an adult: A case report and review of the literature. World J Gastrointest Pathophysiol 1(5): 171-176.

3. Verdin V, Seydel B, Detry O (2010) Le lymphangiome kystique du mésentère. Rev Med Liège 65(11): 615-618.

4. Chegdali O, Guedira M, Benaddi L (2004) Lymphangiome kystique abdominal de ladulte. Acta Endosc 34: 245-248.

5. Sadick M, Müller Wille R, Wildgruber Met, Wohlgemuth WA (2018) Vascular anomalies (Part I): Classification and diagnostics of vascular anomalies. Rofo 190(9): 825-835.

6. Bezzola T, Bülher L, Chardot C (2008) Le traitement chirurgical du lymphangiome kystique abdominal chez ladulte et chez lenfant. J Chir 145(3): 238-243.

7. Losanoff JE, Kjossev KT (2005) Mesenteric cystic lymphangioma : Unusual cause of intra-abdominal catastrophe in an adult. Int J Clin Pract 59(8): 986-987.

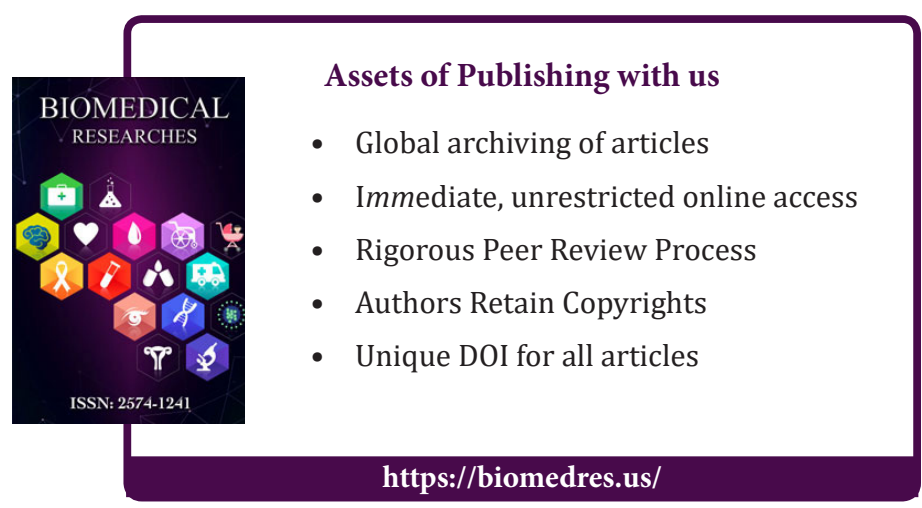

